\title{
Author Correction: Complex genetic signatures in immune cells underlie autoimmunity and inform therapy
}

Valeria Orrù (1), Maristella Steri (1D, Carlo Sidore (D), Michele Marongiu (D), Valentina Serra, Stefania Olla, Gabriella Sole, Sandra Lai, Mariano Dei, Antonella Mulas, Francesca Virdis, Maria Grazia Piras, Monia Lobina (D), Mara Marongiu, Maristella Pitzalis, Francesca Deidda, Annalisa Loizedda, Stefano Onano, Magdalena Zoledziewska, Stephen Sawcer, Marcella Devoto, Myriam Gorospe, Gonçalo R. Abecasis (D), Matteo Floris @iD, Mauro Pala, David Schlessinger, Edoardo Fiorillo and Francesco Cucca (i)

Correction to: Nature Genetics https://doi.org/10.1038/s41588-020-0684-4, published online 14 September 2020.

In the version of this article initially published, in the second sentence of the Abstract, 'independent association signals for 459 cell traits at 69 loci ( 52 of them novel)' should have read 'independent association signals for 459 cell traits at 70 loci ( 53 of them novel)' to match the correct values in the main text. The error has been corrected in the HTML and PDF versions of the article.

Published online: 18 September 2020

https://doi.org/10.1038/s41588-020-00718-6

( ) The Author(s), under exclusive licence to Springer Nature America, Inc. 2020

\section{Author Correction: An integrated multi-omics approach identifies epigenetic alterations associated with Alzheimer's disease}

Raffaella Nativio, Yemin Lan, Greg Donahue, Simone Sidoli (D), Amit Berson, Ananth R. Srinivasan, Oksana Shcherbakova (D), Alexandre Amlie-Wolf(D), Ji Nie, Xiaolong Cui, Chuan He DD, Li-San Wang, Benjamin A. Garcia, John Q. Trojanowski (D), Nancy M. Bonini (D) and Shelley L. Berger (D)

Correction to: Nature Genetics https://doi.org/10.1038/s41588-020-0696-0, published online 28 September 2020.

In the version of this article initially published, in the Acknowledgements, the name of the Kleberg Foundation was incorrectly spelled as the Kleber foundation. The error has been corrected in the HTML and PDF versions of the article.

Published online: 8 October 2020

https://doi.org/10.1038/s41588-020-00733-7

(c) The Author(s), under exclusive licence to Springer Nature America, Inc. 2020 